\title{
Electrochemical preparation of a rugate filter in silicon and its deviation from the ideal structure
}

$\operatorname{AUTHOR}(\mathrm{S}):$

Salem, MS; Sailor, MJ; Sakka, T; Ogata, YH

\section{CITATION:}

Salem, MS ... [et al]. Electrochemical preparation of a rugate filter in silicon and its deviation from the ideal structure. JOURNAL OF APPLIED PHYSICS 2007, 101(6): 063503.

\section{ISSUE DATE:}

2007-03-15

URL:

http://hdl.handle.net/2433/50431

\section{RIGHT:}

Copyright 2007 American Institute of Physics. This article may be downloaded for personal use only. Any other use requires prior permission of the author and the American Institute of Physics. 


\title{
Electrochemical preparation of a rugate filter in silicon and its deviation from the ideal structure
}

\author{
M. S. Salem \\ Institute of Advanced Energy, Kyoto University, Uji, Kyoto 611-0011, Japan \\ M. J. Sailor \\ Department of Chemistry and Biochemistry, University of California at San Diego, \\ San Diego, California 92039-0358
}

\author{
T. Sakka and Y. H. Ogata ${ }^{a}$ \\ Institute of Advanced Energy, Kyoto University, Uji, Kyoto 611-0011, Japan
}

(Received 19 December 2006; accepted 10 January 2007; published online 16 March 2007)

\begin{abstract}
Porous silicon single layers are formed by anodization of silicon in a hydrofluoric acid solution at different current densities. An accurate estimation of the etch rates and refractive indices is performed. The refractive index-depth profile of a porous silicon rugate filter (PSRF) prepared by sinusoidally modulating the current density during etch is investigated. The produced PSRF is found to have a periodic pseudo-sinusoidal variation of refractive index with depth. The sinusoidal current density waveform that was employed generates a nonideal refractive index-depth profile with an asymmetric period. It is found that this deformed refractive index profile affects the quality of the characteristic resonant peak of the rugate filter. A simple modification of the current density waveform can be implemented, which allows one to achieve a true sinusoidal refractive index-depth profile characteristic of a rugate filter. The modified current density waveform allows the generation of a PSRF with a low index contrast and a sharp resonant band. The ability of these structures to act as chemical vapor sensors is tested and compared with the existing literature. The filters exhibit a red-shift in the photonic feature upon exposure to saturated ethanol vapor. The modified signal does not impose a significant change on the filter sensitivity to ethanol vapor. (C) 2007 American Institute of Physics. [DOI: 10.1063/1.2710339]
\end{abstract}

\section{INTRODUCTION}

Porous silicon is created by electrochemical anodization of silicon in a hydrofluoric acid solution. ${ }^{1}$ It exhibits a great potential for use in chemical and biological sensing, ${ }^{2}$ optical switching devices, ${ }^{3}$ implantable biomaterials, ${ }^{4}$ and in highthroughput screening applications. ${ }^{5}$ The refractive index of this material is dependent on its porosity, which is the volumetric fraction of pores in the structure. Electrochemical dissolution of silicon in hydrofluoric acid solution proceeds preferentially at the pore bottom. ${ }^{6}$ This implies that the already established porous layer persists during further electrochemical etching. Therefore, almost any desired porosity profile can be achieved by changing the applied current during etch. ${ }^{7}$ By this means, a porous silicon multilayer with a characteristic photonic band can easily be formed.

In comparison with porous silicon single layers, the reflectance of multilayer structures can reach values of up to 99.5\% which strongly exceeds the reflectance of crystalline silicon. ${ }^{8}$ The increased reflectance arises from multiple reflection and interference that occurs in the multilayer due to the modulated index contrast. The strong modulation also offers an elegant way to narrow the broad photoluminescence band of porous silicon. ${ }^{9}$ The spectral peak position of the multilayer is a strong function of the average refractive

\footnotetext{
a) Author to whom correspondence should be addressed; electronic mail: y-ogata@iae.kyoto-u.ac.jp
}

indices of the layers, and a shift in the photonic feature has been shown to provide a very sensitive method for detection of condensable vapors ${ }^{10,11}$ and other molecules that can enter the pores. This method can also be applied for the detection of explosives ${ }^{12}$ and chemical weapons. ${ }^{13}$ Etching of porous silicon multilayers, on the other hand, provides a simple means to fabricate interference filters ${ }^{14}$ and optical waveguides $^{15}$ onto pure silicon wafers. Furthermore, the electrochemical fabrication process is very compatible with microelectromechanical systems (MEMS)/microoptoelectromechanical systems (MOEMS) compared to the conventional methods used to prepare optical filters. ${ }^{16}$

The formation of porous silicon multilayers can be carried out either by using a periodically doped substrate or by varying the etching parameters, such as current density, during the anodization process. The former approach is less convenient compared to the latter because of the need for an epitaxially grown wafer. ${ }^{17} \mathrm{~A}$ discrete variation of current density between two values gives rise to the formation of a Bragg stack provided that the optical thickness of each layer in the stack is a quarter of the resonant wavelength. ${ }^{18}$ Porous silicon Bragg mirrors were first prepared by Vincent. ${ }^{19} \mathrm{~A}$ continuous modulation of the current density can also be employed to establish a structure with a continuous refractive index profile, as required for a rugate filter. ${ }^{20}$ This filter is one of a class of optical filters that possess a sinusoidal variation of the refractive index in a direction perpendicular to the surface. The first silicon-based rugate filter fabricated 
by the electrochemical technique was accomplished by Berger et al. $^{21}$ A porous silicon rugate filter (PSRF) is often preferred over the conventional Bragg stack because it does not display higher order harmonics. ${ }^{22}$ In addition, the individual layers of Bragg filters must be designed such that they are phase-matched, while rugate filters do not have this requirement and so they are simpler to fabricate. ${ }^{23}$

In the electrochemical design of a PSRF, it is generally assumed that the sinusoidal variation of the etching current with time maps to a corresponding similar sinusoidal change of refractive index with depth because porosity and, therefore, refractive index of the formed porous layer vary with current density. ${ }^{24}$ This assumption is reasonable as long as the etching rate is a constant function of current density. Herein, we investigate the dependence of etching velocity on the current density and its related effects that should be taken into consideration during the design of PSRFs. Our starting substrate was lightly doped silicon $(p-\mathrm{Si})$. This substrate is commonly less preferred than heavily doped silicon $\left(p^{+}-\mathrm{Si}\right)$ since its resulting multilayer is mechanically more fragile and more difficult to process. However, a substrate with a low doping level leads to the formation of a high surfacearea, microporous structure that is more appropriate for the sensing of low concentrations of chemical vapors. ${ }^{25}$ Furthermore, porous silicon formed from $p$-Si substrates have a smaller free carrier absorption and they display much brighter luminescence compared to $p^{+}-\mathrm{Si}^{26}$

\section{EXPERIMENTAL}

Several porous silicon layers were formed by electrochemical anodization of a (100)-oriented boron-doped $p$-type silicon wafer $(3.2-4.8 \Omega \mathrm{cm}$ in resistivity) in a $1: 0.6(\mathrm{v} / \mathrm{v})$ solution of $49 \%$ aqueous hydrofluoric acid and ethanol at various current densities. Aluminum was evaporated onto the back side of the silicon wafer to ensure a good ohmic contact. The current was supplied by a programmable current source (Keithly 6221). Prior to anodization, the silicon wafer was dipped in 5 wt. \% HF solution to remove the native oxide. An electrochemical anodization cell made of trifluoroethylene resin was employed with an O-ring at the bottom that allowed an effective electrode area of $0.79 \mathrm{~cm}^{2}$. After anodization, the sample was dried under a gentle stream of argon gas. Thickness measurements of the resulting layers were carried out by a JSM-5600 JEOL scanning electron microscope (SEM). The porosity of some selected porous silicon layers was determined gravimetrically. The samples were weighed on a laboratory balance with a resolution of $10 \mu \mathrm{g}$. Samples were weighed firstly before the etching process, then after porosification, and finally after removing the porous layer in a $3 \mathrm{wt}$. \% $\mathrm{KOH}$ solution. A PSRF was prepared using the same HF solution using a sinusoidal current density oscillating between 115.3 and $10.2 \mathrm{~mA} \mathrm{~cm}^{-2}$. The period of the sinusoidal current waveform was $4.7 \mathrm{~s}$ with a repetition of 100 cycles.

Interferometric reflectance spectra of the prepared samples were recorded using a charge-coupled device (CCD) spectrometer (Ocean Optics HR-4000CG-UV-NIR). A tungsten halogen lamp was used as a source of illumination.

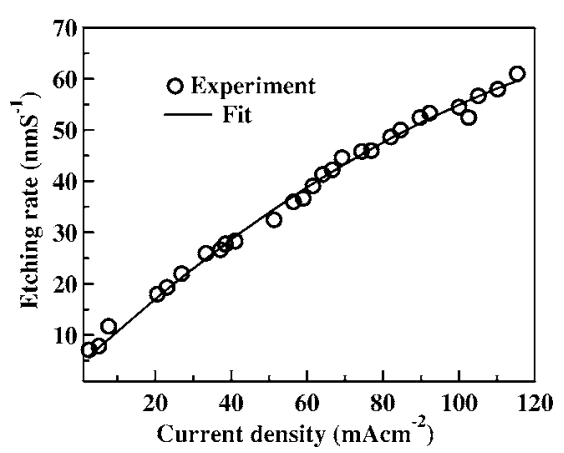

FIG. 1. Etching rate of lightly doped silicon substrate in a 1:0.6 (v/v) of $49 \%$ aqueous hydrofluoric acid and ethanol as a function of current density.

Spectra were collected with the CCD detector in the wavelength range 400-1200 $\mathrm{nm}$ and corrected for the spectral response of the lamp.

\section{RESULTS AND DISCUSSION}

The correlation between the optical constants and electrochemical formation conditions is indispensable for accurate design of a PSRF. For this sake, porous silicon layers were prepared using various current densities ranging from 2.5 to $115 \mathrm{~mA} \mathrm{~cm}^{-2}$. The etch rate was determined by a measurement of the thickness of the porous silicon after etching using cross-sectional SEM images. Figure 1 shows the variation of the etching rate of silicon as a function of the anodizing current density. The etching rate rises steadily as the current density increases. A power function is adopted to fit the monotonic increase of the etching rate with the current density. ${ }^{27}$

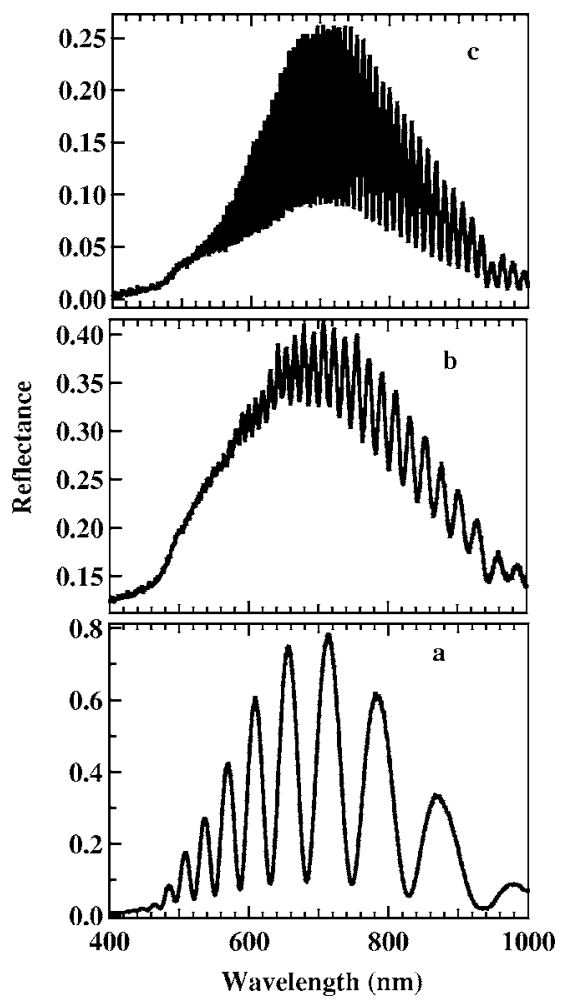

FIG. 2. Reflectance spectra of three porous silicon layers formed at (a) 2.5, (b) 33, and (c) $99 \mathrm{~mA} \mathrm{~cm}^{-2}$. The anodization time is $5 \mathrm{~min}$. 


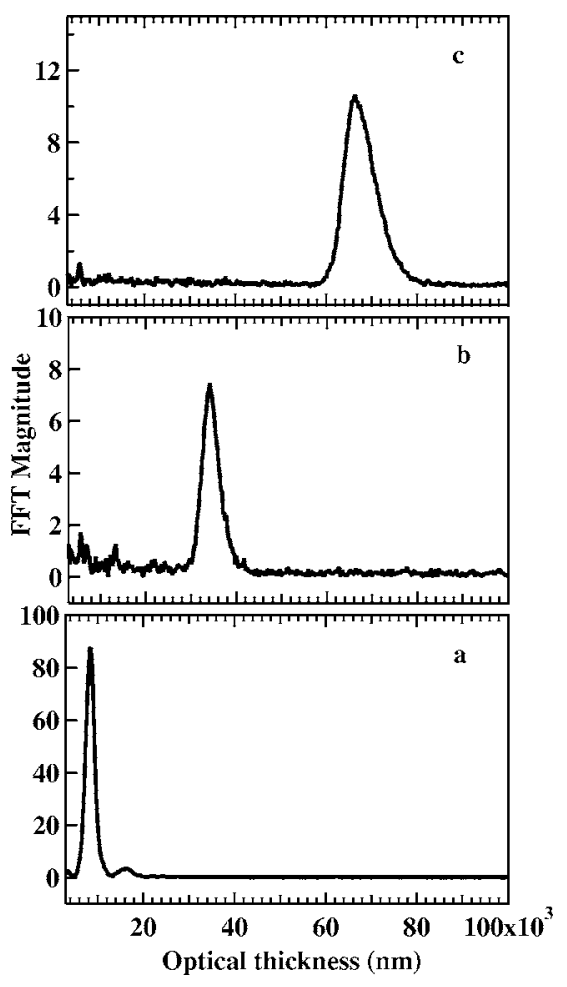

FIG. 3. FFT of the reflectance spectra displayed in Fig. 2 and formed at (a) 2.5 , (b) 33 , and (c) $99 \mathrm{~mA} \mathrm{~cm}^{-2}$. The anodization time is $5 \mathrm{~min}$.

The reflectance spectrum of individual porous silicon layers was recorded to estimate the corresponding optical parameters. All samples were prepared at a constant anodization time of $5 \mathrm{~min}$. The results are shown in Fig. 2 for three porous silicon samples prepared at $2.5,33,99 \mathrm{~mA} \mathrm{~cm}^{-2}$. Each spectrum displays a remarkable series of interference fringes. These fringe patterns result from the interference of light coming from the porous silicon top surface and its interface with the silicon substrate (Fabry-Pérot interference). The reflectance of the samples decreases with an increase in the current density used in preparation. Higher current densities produce highly porous layers. The higher air content results in lower refractive index, and hence lower reflectance according to effective medium theories. ${ }^{28}$ The higher current density also results in significant roughening of the film, ${ }^{29,30}$ and the interface roughness of the porous silicon sample prepared at a current density of $99 \mathrm{~mA} \mathrm{~cm}{ }^{-2}$ results in suppression of the interference fringes. However, well-defined fringes were still observed in its reflectance spectrum.

A simple and convenient method of extracting the optical parameters is based on the fast Fourier transform (FFT) of the reflectance spectrum. ${ }^{31}$ The FFT can provide reliable optical constants especially for complicated structures. ${ }^{32}$ In order to get the Fourier transform of the spectrum, the wavelength axis was inverted and a linear interpolation was applied such that the reflectance data were evenly spaced in units of wavenumbers $\left(\mathrm{nm}^{-1}\right)$. FFT of the reflectance spectra of Fig. 2 are presented in Fig. 3. Each series of interference fringes produces a single peak in the Fourier transform. The intensity of Fourier transforms is proportional to the refractive index contrast of the film, relative to its neighboring layers. The value of the optical thickness can be directly

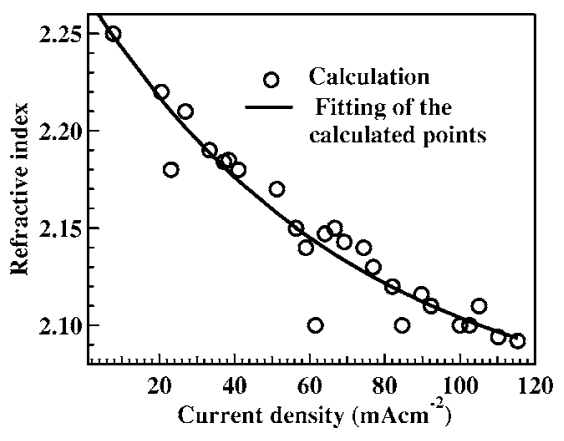

FIG. 4. Refractive index estimated from the FFT peak position as a function of current density. The optical thickness can be estimated as half of the peak position.

calculated as half of the position of the resulting peak. The refractive index was obtained by dividing the optical thickness obtained from the FFT by the physical thickness. The refractive index values estimated from this method were compared to that obtained from the position of interference fringes. We found good agreement between both methods. The variation of the refractive index with current density is depicted in Fig. 4. In this figure, it is clear that the refractive index does not change so much by changing current density over a wide range. This fact is common for porous silicon layers fabricated from lightly doped silicon substrates. ${ }^{21}$ The refractive index, on the other hand, can be linked to the porosity, $P_{\text {in }}$, using Bruggeman's effective-mediumapproximation $^{28}$ given by

$$
P_{\text {in }} \frac{n_{\text {air }}^{2}-n_{\text {eff }}^{2}}{n_{\text {air }}^{2}+2 n_{\text {eff }}^{2}}+\left(1-P_{\text {in }}\right) \frac{n_{\mathrm{Si}}^{2}-n_{\text {eff }}^{2}}{n_{\mathrm{Si}}^{2}+2 n_{\text {eff }}^{2}}=0,
$$

where $n_{\text {air }}, n_{\mathrm{Si}}$, and $n_{\text {eff }}$ are the refractive indices of air, silicon, and the effective porous medium, respectively. This model is simple and applicable to porous materials which have moderate porosity, though it neglects optical absorption effects. We measured the porosity of some selected porous silicon layers. The corresponding refractive index was calculated from relation (1) and compared to that obtained by the FFT method. Results are summarized in Table I. The refractive index calculated by the effective medium model is smaller than that provided by the FFT analysis. This deviation might be attributed to optical absorption effects, for which the classical Bruggeman model is not completely valid.

The calibration curves of the etching rate and measured refractive index as a function of current density were employed to investigate the actual refractive index-depth profile

TABLE I. Porosity and refractive index determination at different current densities.

\begin{tabular}{cccc}
\hline \hline $\begin{array}{c}\text { Current density } \\
\left(\mathrm{mA} \mathrm{cm}^{-2}\right)\end{array}$ & $\begin{array}{c}\text { Porosity } \\
(\%)\end{array}$ & $\begin{array}{c}\text { Refractive index } \\
\text { estimated by } \\
\text { the Bruggeman model }\end{array}$ & $\begin{array}{c}\text { Refractive index } \\
\text { determined by } \\
\text { the FFT method }\end{array}$ \\
\hline 21 & 53 & 2.10 & 2.21 \\
32 & 58 & 1.94 & 2.19 \\
58 & 70 & 1.58 & 2.14 \\
108 & 78 & 1.37 & 2.09 \\
\hline
\end{tabular}




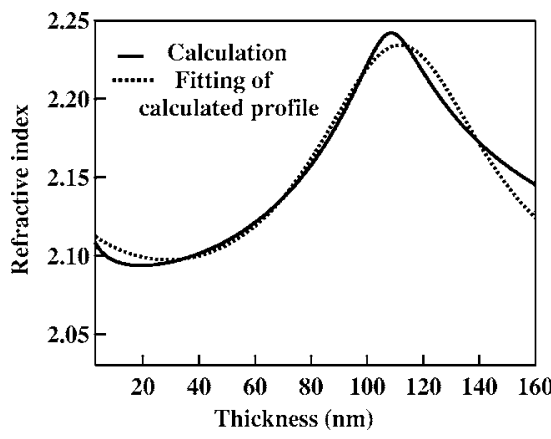

FIG. 5. Calculated (solid line) and fitted (dashed line) refractive index-depth profiles using Eq. (2) corresponding to one cycle of a sinusoidal current density oscillating between 115.3 and $10.2 \mathrm{~mA} \mathrm{~cm}^{-2}$.

associated with the sinusoidal variation of current density. If we apply a sinusoidal current density oscillating, for example, between 115.3 and $10.2 \mathrm{~mA} \mathrm{~cm}^{-2}$, the corresponding refractive index modulation does not take the same sinusoidal shape. It shows instead a deformed profile with an asymmetric period displayed in Fig. 5. The obtained asymmetric cycle was fitted to the equation

$$
n(x)=A+\frac{B}{C+D \sin [p(x+E)]},
$$

where $p$ is the periodicity and $A, B, C, D$, and $E$ are the fitting parameters. This is just a mathematical function that can fit well the pseudo-asymmetric shape, and hence these parameters do not include physical meaning. However, the adjustment of these parameters gives a best fit to the asymmetric sinusoidal shape. The appearance of the nonsinusoidal index profile is ascribed to the variation of the etching rate with the current density. This variation can be easily taken into account during the preparation of Bragg stacks because of their discrete method of formation. However, in all of the previous works, no special attention was paid to the actual variation of the refractive index-depth profile of PSRFs. This modulation has been generally assumed to map directly to the applied sinusoidal etch current density. ${ }^{33}$ This implies that the etching rate is a constant function of the current density. A uniform etching rate cannot be guaranteed during the preparation, especially when we span a wide range of current density to have a large porosity gradient.

According to the periodicity and limiting values of the sinusoidal current density, the refractive index-depth profile might assume a deformed nonsinusoidal shape. Figure 6 displays one cycle of refractive index profiles deviating from the ideal sinusoidal shape and oscillating between $n=2.04$ and 2.30. Trace a represents an ideal sinusoidal shape. Traces $\mathrm{b}$ and $\mathrm{c}$ were approximated by relation (2) by adjusting its parameters $A, B, C, D$, and $E$ as $2,0.3,5.3,4.1$, and 17, respectively, in the case of trace b and 2, 3, 915, 904, and 27, respectively, for trace c. The conditions for trace c give higher deformation than that for trace $b$. The corresponding reflectance spectra representing a repetition of 50 cycles are theoretically calculated as depicted in Fig. 7. The theoretical reflectance spectrum was calculated based on the transfer matrix method. ${ }^{34}$ The continuous variation of refractive index was represented as a large number of discrete layers. We

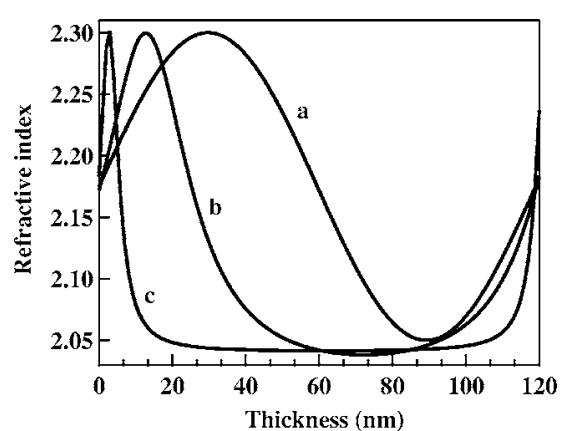

FIG. 6. Estimated refractive index-depth profiles for one cycle of a sinusoidal current density waveform, showing the distortion produced by the nonlinear mapping of current density to refractive index. Trace a shows the sinusoidal period. Traces $\mathrm{b}$ and $\mathrm{c}$ display some deviations from the sinusoidal shape. Traces $\mathrm{b}$ and $\mathrm{c}$ were plotted using Eq. (2) by adjusting its parameters $A, B, C, D$, and $E$ as $2,0.3,5.3,4.1$, and 17 , respectively, in the case of trace b and 2, 3, 915, 904, and 27, respectively, for trace c.

divided each cycle of the refractive index profile into different numbers of very thin discrete layers and calculated the reflectance spectrum in each case until the result was independent of the number of layers. We found that dividing each cycle of the index profile into more than 60 layers has no effect on the calculated spectrum. The dotted curves of Fig. 7 represent the calculated reflectance spectra, taking into account the effect of underlying silicon substrate dispersion and neglecting the effect of absorption. The sinusoidal refractive index profile shown in Fig. 6(a) produces a stop band centered at $510 \mathrm{~nm}$ with a full width at half maximum (FWHM) of $20 \mathrm{~nm}$ as displayed in Fig. 7(a). A slight deviation from the sinusoidal profile shown in Fig. 6(b) does not produce significant change in the corresponding reflectance spectrum depicted in Fig. 7(b). When the refractive index profile becomes more asymmetric, the reflectance spectrum gets sharper, and a slight decrease in intensity is predicted. The obtained blue-shift of the reflectance spectrum can be

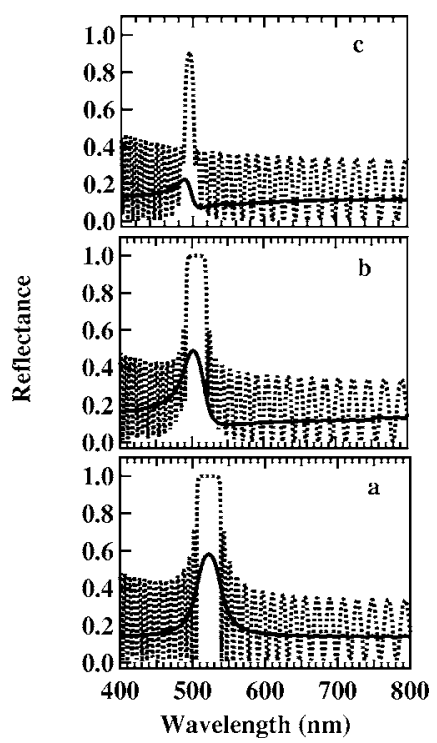

FIG. 7. Calculated reflectance spectra of 50-cycle rugate filters having the asymmetric periods. Curves $\mathrm{a}, \mathrm{b}$, and $\mathrm{c}$ indicate its corresponding refractive index traces shown in Fig. 6. The dotted curves neglect optical absorption effects, whereas the solid curves account for the effects of absorption by the multilayer structure. 


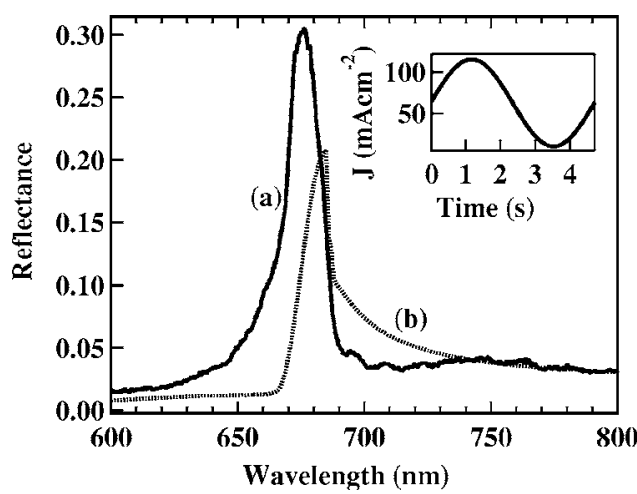

FIG. 8. Measured (a, solid line) and calculated (b, dashed line) reflectance spectra for a 100-cycle PSRF formed using a sinusoidal current density waveform (a single cycle is shown in the inset). The refractive index profile used in the calculation is derived from the current density-refractive index profile displayed in Fig. 4.

attributed to the decrease of the average optical thickness of the filter $\int_{0}^{p} n(x) d x$ as the profile becomes asymmetric. The average refractive index of the pseudo-sinusoidal profile is decreased by its phase shift with the sinusoidal profile. The sharpness of the spectrum is a strong function of the limiting values of the refractive index and the number of repeats in the structure. The width of the reflectance band $(\Delta \lambda)$ is linked to the optical admittance ratio by the relation ${ }^{20}$

$$
\frac{\Delta \lambda}{\lambda}=\frac{1}{2} \ln \frac{\eta_{M}}{\eta_{m}},
$$

where $\lambda$ is the resonant wavelength of the filter, and $\eta_{M}$ and $\eta_{m}$ are the lower and upper values of the refractive index profile, respectively. If the spectrum is blue-shifted by increasing the thickness of the entire filter, the width of the spectrum decreases. ${ }^{35}$ This is consistent with Eq. (3). The same approach can be used to explain the narrowing of the reflectance band observed as the refractive index profile becomes asymmetric. Optical absorption was introduced into the model as a wavelength-independent extinction coefficient of 0.04 . When the effect of absorption is included into the calculations as shown in the solid curves of Fig. 7, the magnitude of reflectance shows a significant decrease and the spectrum deteriorates as the profile becomes more asymmetric. This implies that an asymmetric refractive index profile will decrease the intensity and peak width of the stop band, especially for an absorbing structure.

Figure 8 shows the experimental and simulated reflectance spectrum of a PSRF prepared using the sinusoidal current density waveform shown in the inset of the figure. The simulated spectrum was calculated using the actual refractive index profile displayed in Fig. 5. The absorption effect was included into this simulation. The FWHM of the experimental spectrum matches the simulated one. The experimental reflectance spectrum shows a slight blue-shift compared to the corresponding simulated one. This might be attributed to natural oxidation of the freshly prepared filters. Porous silicon prepared from lightly doped silicon air-oxidizes fairly quickly. ${ }^{36}$ Presuming an ideal sinusoidal refractive index profile, the simulated spectrum exhibits a red-shift with a larger FWHM. In our previous study, we observed a similar dis-

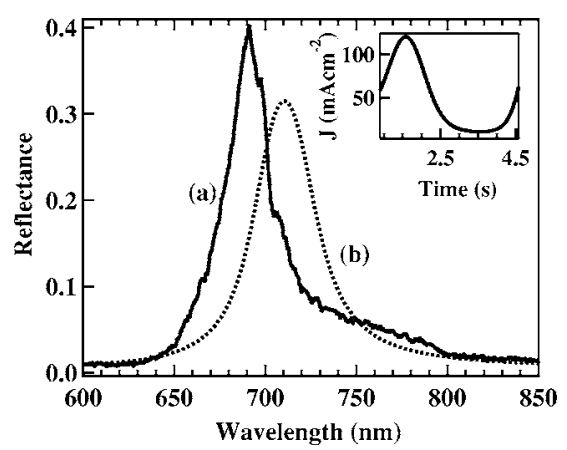

FIG. 9. Reflectance spectrum of a 100-cycle PSRF formed by a modified sinusoidal current density shown in the inset (a) and that simulated by assuming a sinusoidal variation of the refractive index with the depth (b).

crepancy between the experimental data and the theoretical calculations, based on the assumption of an ideal sinusoidal refractive index profile. ${ }^{37}$ It should be noted that the intensity of the simulated reflectance in Figs. 8 and 9 is lower than the experimental one. This can be attributed to the deviation of the calculated extinction coefficient from its actual value. The actual extinction coefficient would be lower than the calculated one. This is because the calculated value is taken from the spectrum of a single layer. However, in the case of the multilayer we should describe an effective extinction coefficient for the overall light loss in the multilayer which is supposed to be lower than the case of the single layer due to the reflectance enhancement by multiple reflections and interference of light expected from the multilayer structure.

The etching current density waveform was modified as shown in the inset of Fig. 9. In this figure, the experimental and simulated reflectance spectra are displayed. The FWHM of the experimental spectrum is comparable to the simulated one. The modified current density produces a true sinusoidal refractive index profile according to the empirical calibration curves relating refractive index and etching rate. The modi-

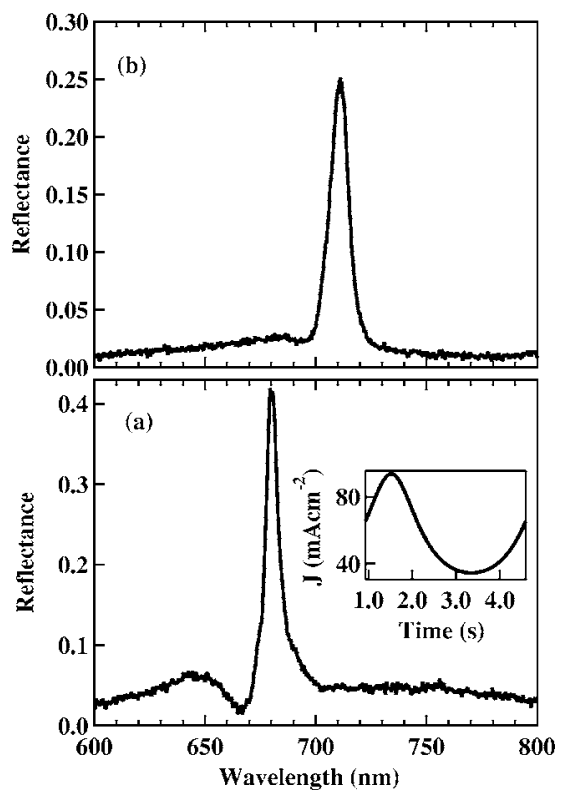

FIG. 10. Reflectance spectrum of a PSRF prepared by the modified current waveform shown in the inset (a) and that produced after exposing the filter to saturated ethanol vapor (b). 
fied current density waveform displayed in the inset of Fig. 10(a) was employed to prepare a PSRF. This signal has a lower peak to peak current value compared to that shown in Fig. 9. This low current modulation produces a low index contrast and subsequently a sharp resonant band. The collected reflectance spectrum of this sample has a FWHM of $\sim 10 \mathrm{~nm}$ as shown in Fig. 10(a). When this structure is exposed to saturated ethanol vapor in nitrogen for $10 \mathrm{~min}$, a remarkable red-shift is obtained as shown in Fig 10(b). This shift represents an increase of the average refractive index of the filter, and can be attributed to microcapillary condensation of ethanol vapor into the nanometer-sized pores. ${ }^{38} \mathrm{~A}$ structure prepared using a simple sinusoidal current density waveform displays the same sensitivity to ethanol vapor. Thus the improvement in the quality of the spectral feature does not have a significant effect on the absolute spectral shift experienced by the nanostructure. However, because the line width is significantly smaller for the samples etched using the modified current density waveform, a microsensor system based on a narrow linewidth $\mathrm{LED}^{39}$ or laser probe ${ }^{40}$ is expected to show improved sensitivity.

\section{CONCLUSIONS}

The relationship between the etching rate and current density is very important to consider in designing PSRFs based on electrochemical etching of silicon. Due to the increase of the etch rate with the current density, the sinusoidal modulation of the current density produces a pseudosinusoidal refractive index profile with an asymmetric period. The reflectance spectrum associated with this profile exhibits a narrower resonant band with a lower intensity compared to the sinusoidal refractive index modulation. As the profile becomes more asymmetric, the resonant band disappears especially in the case of an absorbing filter. A modification of the sinusoidal current modulation seems to be useful to have an accurate filter design employed for practical applications.

\section{ACKNOWLEDGEMENTS}

This work was partially supported by a grant-in-aid from the Japan Society for the Promotion of Science under Grant No. 18350107. One of the authors (M.J.S.) was supported by the National Science Foundation under Grant No. DMR 0452579 .

\footnotetext{
${ }^{1}$ A. Uhlir, Bell Syst. Tech. J. 35, 333 (1956).

${ }^{2}$ H. Sohn, S. Létant, M. J. Sailor, and W. C. Trogler, J. Am. Chem. Soc. 122, 5399 (2000)

${ }^{3}$ V. Lehmann, R. Stengl, H. Reisinger, R. Detemple, and W. Theiss, Appl. Phys. Lett. 78, 589 (2001).

${ }^{4}$ L. T. Canham, M. P. Stewart, J. M. Buriak, C. L. Reeves, M. Anderson, E. K. Squire, P. Allcock, and P. A. Snow, Phys. Status Solidi A 182, 521 (2000).
}

${ }^{5}$ F. Cunin, T. A. Schmedake, J. R. Link, Y. Y. Li, J. Koh, S. N. Bhatia, and M. J. Sailor, Nat. Mater. 1, 39 (2002).

${ }^{6}$ I. Ronga, A. Bsiesy, F. Gaspard, R. Hérino, M. Ligeon, F. Muller, and A. Halimaoui, J. Electrochem. Soc. 138, 1403 (1991).

${ }^{7}$ S. O. Meade, M. S. Yoon, K. H. Ahn, and M. J. Sailor, Adv. Mater. (Weinheim, Ger.) 16, 1811 (2004).

${ }^{8}$ S. Setzu, S. Létant, P. Solsona, R. Romestain, and J. C. Vial, J. Lumin. 80, 129 (1998).

${ }^{9}$ L. Tsybeskov, Ju. V. Vandyshev, and P. M. Fauchet, Phys. Rev. B 49, 7821 (1994).

${ }^{10}$ P. A. Snow, E. K. Squire, P. St. J. Russell, and L. T. Canham, J. Appl. Phys. 86, 1781 (1999).

${ }^{11}$ T. Gao, J. Gao, and M. J. Sailor, Langmuir 18, 9953 (2002).

${ }^{12}$ S. Content, W. C. Trogler, and M. J. Sailor, Chem.-Eur. J. 6, 2205 (2000).

${ }^{13}$ H. Sohn, S. Letant, M. J. Sailor, and W. C. Trogler, J. Am. Chem. Soc. 122, 5399 (2000).

${ }^{14}$ V. Pellegrini, A. Tredicucci, C. Mazzoleni, and L. Pavesi, Phys. Rev. B 52, R14328 (1995)

${ }^{15}$ S. Nagata, C. Domoto, T. Nishimura, and K. Iwameji, Appl. Phys. Lett. 72, 2945 (1998).

${ }^{16}$ D. Hunkel, M. Marso, R. Butz, R. Arens-Fisher, and H. Lüth, Mater. Sci. Eng., B 69-70, 100 (2000).

${ }^{17}$ St. Frohnhoff, M. G. Berger, M. Thönissen, C. Dieker, L. Vescan, H. Münder, and H. Lüth, Thin Solid Films 255, 59 (1995).

${ }^{18}$ H. A. Lopez and P. M. Fauchet, Appl. Phys. Lett. 77, 3704 (2000).

${ }^{19}$ G. Vincent, Appl. Phys. Lett. 64, 2367 (1994).

${ }^{20}$ B. G. Bovard, Appl. Opt. 32, 5427 (1993).

${ }^{21}$ M. G. Berger, R. Arens-Fischer, M. Thönissen, M. Krüger, S. Billat, H. Lüth, S. Hilbrich, W. Theiß, and P. Grosse, Thin Solid Films 297, 237 (1997).

${ }^{22}$ M. Thönissen, M. G. Berger, W. Theiss, S. Hilbrich, M. Krüger, and H. Lüth, Solid State Phenom. 45, 65 (1997).

${ }^{23}$ J. R. Dorvee, A. M. Derfus, S. N. Bhatia, and M. J. Sailor, Nat. Mater. 3, 896 (2004)

${ }^{24}$ E. Lorenzo, C. J. Oton, N. E. Capuj, M. Ghulinyan, D. Navarro-Urrios, Z. Gaburro, and L. Pavesi, Phys. Status Solidi C 2, 3227 (2005).

${ }^{25}$ M. S. Salem, M. J. Sailor, F. A. Harraz, T. Sakka, and Y. H. Ogata, Phys. Status Solidi C (in press).

${ }^{26}$ S. Setzu, P. Ferrand, and R. Romestain, Mater. Sci. Eng., B 69-70, 34 (2000).

${ }^{27}$ E. Haimi, V. K. Lindroos, and R. Nowak, J. Nanosci. Nanotechnol. 1, 201 (2001).

${ }^{28} \mathrm{~W}$. Theiss, in Festkörperprobleme/Advances in Solid State Physics, Vol. 33, edited by R. Helbig (Vieweg, Braunschweig, 1994), p.149.

${ }^{29}$ S. Setzu, G. Lérondel, and R. Romestain, J. Appl. Phys. 84, 3129 (1998).

${ }^{30}$ usG. Lérondel, R. Romestain, and S. Barret, J. Appl. Phys. 81, 6171 (1997).

${ }^{31}$ C. Pacholski, M. Sartor, M. J. Sailor, F. Cunin, and G. M. Miskelly, J. Am. Chem. Soc. 127, 11636 (2005).

${ }^{32}$ C. Pacholski, C. Yu, G. M. Miskelly, D. Godin, and M. J. Sailor, J. Am. Chem. Soc. 128, 4250 (2006).

${ }^{33}$ S. Ilyas, T. Böcking, K. Kilian, P. J. Reece, J. Gooding, K. Gaus, and M. Gal, Opt. Mater. (Amsterdam, Neth.) 29, 619 (2007).

${ }^{34}$ M. Born and E. Wolf, Principle of Optics (Pergamon, Oxford, 1980).

${ }^{35}$ M. Thönissen, M. G. Berger, S. Billat, R. Arens-Fischer, M. Krüger, H. Lüth, W. Theiß, S. Hillbrich, P. Grosse, G. Lerondel, and U. Frotscher, Thin Solid Films 297, 92 (1997).

${ }^{36}$ W. Theiß, M. Arntez, S. Hilbrich, M. Wernke, R. Arens-Fischer, and M. G. Berger, Phys. Status Solidi B 190, 15 (1995).

${ }^{37}$ M. S. Salem, M. J. Sailor, F. A. Harraz, T. Sakka, and Y. H. Ogata, J. Appl. Phys. 100, 083520 (2006).

${ }^{38}$ S. J. Gregg and K. S. W. Sing, Adsorption, Surface Area and Porosity, 2nd ed. (Academic Press, London, 1982), p. 112.

${ }^{39}$ J. Dorvee and M. J. Sailor, Phys. Status Solidi A 202, 1619 (2005).

${ }^{40}$ J. Gao, T. Gao, and M. J. Sailor, Appl. Phys. Lett. 77, 901 (2000). 